\title{
HETEROGENEIDAD ESPACIAL EN LA DISTRIBUCION DE LOS MACROINVERTEBRADOS A LO LARGO DE UN TRANSECTO EN EL RIO LLOBREGAT (Barcelona, España)
}

\author{
I. Muñoz, N. Prat, X. Millet, E. Martínez Ansemil* \\ Dpto. Ecología. Facultad de Biología. Universidad de Barcelona. \\ *Colegio Universitario de La Coruña.
}

Palabras clave: macroinvertebrates, transect, heterogeneity

\author{
ABSTRACT \\ SPATIAL HETEROGENEITY IN THE MACROINVERTEBRATE DISTRIBUTION ALONG A \\ TRANSECT IN THE LLOBREGAT RIVER (Barcelona, Spain)
}

\begin{abstract}
A stream transect was made in the Llobregat river in Catalonia (NE Spain) taking surber samples. At the sampling location the river was characterized by a patchy substratum of bedrock with Cladophora, macrophytes (Potamogeton pectinatus) and sand.

The seventeen sequential sample replicates yield a list of 100 taxa: 24 Chironomidae, 21 Oligochaeta. 17 Ephemeroptera, 5 Trichoptera, 7 Mollusca, and other minor groups.

The species abundance rank correlation matrix was clustered and yielded five groupes. most of which were clearly related to the substratum and related conditions thus: reophilic species clustered, sand and mud Oligochaeta clustered and Chirinomids asociated with Potamogeton clustered as well.

Diversity values calculated with the Shannon Weaver index, heterogeneity and diversity spectra allow us to describe community diversification and spacial heterogeneity. Such spacial heterogeneity should be considered by those who postulate a diversity decreasement with the increasement ofthe river order.
\end{abstract}

\section{INTRODUCCION}

Los estudios en los ambientes fluviales están rodeados de muchas dificultades metodológicas que se agudizan en la descripción de comunidades de macroinvertebrados cuando las condiciones del medio son diversas. La dificultad de recolección de muestras cuantitativas limita la descripción de las comunidades que en muchos casos presentan una gran heterogeneidad espacial.

La estructuración progresiva que sufre el río aguas abajo lleva asociada una diversificación de condiciones: zona de deposición y zona de erosión. que produce un aumento de la heterogeneidad espacial, difícil de reflejar en los muestreos convencionales.

El uso de muestras en transecto para conocer la distribución de especies de macroinvertebrados bentónicos ha sido poco utilizado y sin embargo puede constituir un buen sistema de muestreo para cono-

Linnética 2: 135-145(1986)

O Asociación Española de Limnología, Madrid. Spain cer mejor la heterogeneidad en el ambiente, (Resh, 1975, 1979), así como para poder establecer una descripción sintética del conjunto. Describimos de esta manera la heterogeneidad y estructuración espacial inabordable de otra forma.

Por otro lado los resultados que se obtienen de muestreos muy estratificados o muy estandarizados, impiden la descripción de la mencionada heterogeneidad.

En este trabajo se pretendía conocer mejor la complejidad presente en el río Llobregat a través del estudio de los macroinvertebrados bentónicos presentes a lo largo de un transecto realizado transversalmente en la zona media del rio. Datos previos sobre las comunidades a lo largo del río o sobre la fauna en general del mismo se pueden encontrar en Prat et al., (1982, 1983, 1984); y Millet \& Prat (1984). 

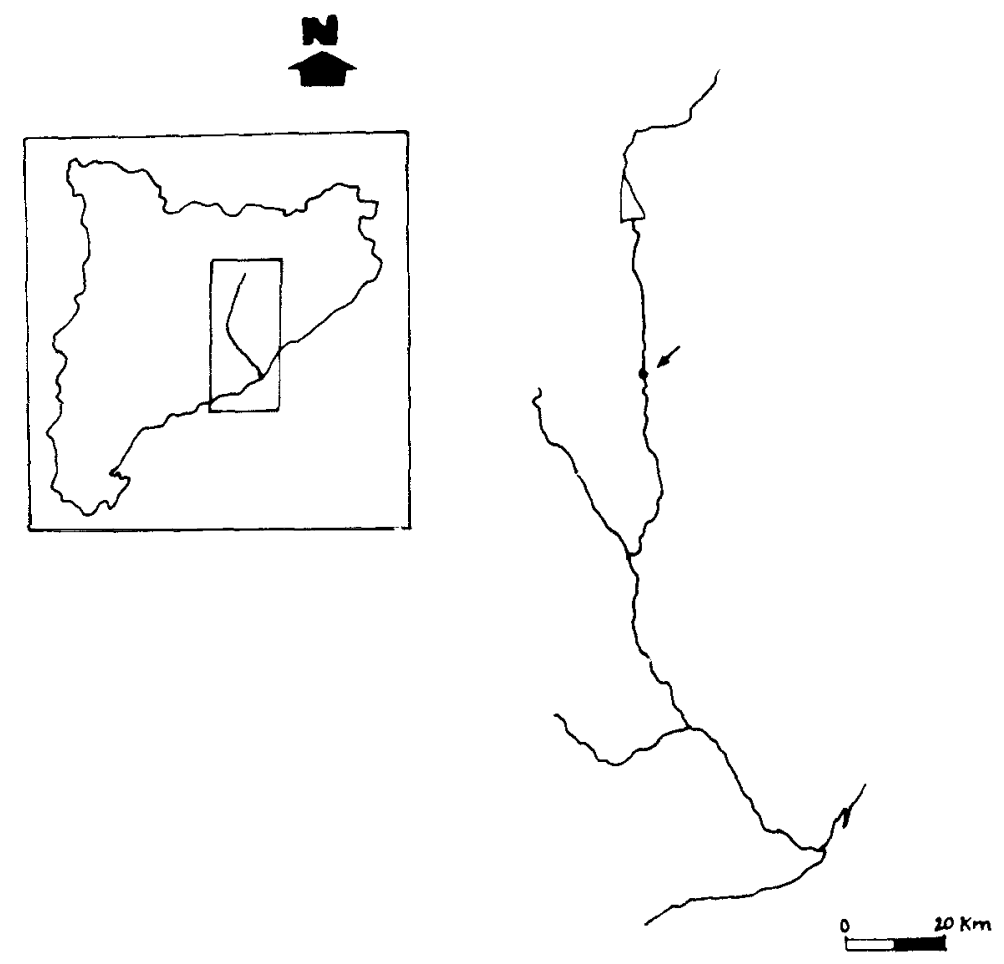

Figura 1.-Situación del río Llobregat y localización del punto de muestreo.

Location of the sampling site.

\section{MATERIAL $Y$ METODOS}

El área muestreada se sitúa en la zona media del eje principal del río Llobregat, entre las localidades de l'Ametlla de Merola y Navars (en la comarca del Bages, provincia de Barcelona, a $\left.41^{\circ} 55^{\prime} \mathrm{N} 1{ }^{\circ} 52^{\prime} \mathrm{E}\right)$. La altura sobre el nivel del mar era de $320 \mathrm{~m}$. y la pendiente media de $0,88 \%$. La distancia al origen del río en este punto es de $53,5 \mathrm{Km}$. (figura 1).

La anchura del río era de $47 \mathrm{~m}$. y la profundidad variaba entre 20 y $77 \mathrm{~cm}$. con una media de 44,86 cm. La sección era en este punto de $21,27 \mathrm{~m}$.' . El caudal en la época de muestreo (5 de agosto de 1982) era de 7,1 m.' $/ \mathrm{sg}$. La velocidad media del agua era de $0,33 \mathrm{~m} . / \mathrm{sg}$.

El lecho del río estaba formado por un sustrato de arena y piedras en proporción variable así como una abundante vegetación macrofítica de Cladophora y Potamogeton pectinatus. Se recogieron 17 muestras de $0,1 \mathrm{~m}$. ${ }^{2}$ de superficie con $2 \mathrm{~m}$. de separación entre ellas a lo largo de un transecto perpendicular al río, de este modo se muestreaban todos los ambientes creados por la interacción entre los elementos que componían el sustrato (figura 2).

Las muestras se recogían utilizando un cuadrado de
$33 \mathrm{~cm}$. de lado. Los materiales desprendidos del sustrato eran colectados en una malla posterior cuyo poro era de $250 \mathrm{um}$, todos los tipos de sustratos eran raspados de forma exhaustiva. Cuando el sustrato lo permitía (arena o barro) era removido con fuerza. Una vez recogidas las muestras se fijaban en el campo con formaldehído al 4\%. En el laboratorio se procedía a la separacion total de la muestra bajo la lupa binocular. Los organismos contados se identificaban hasta el nivel específico siempre que era posible (incluyendo los quironómidos y los oligoquetos, grupos que en los estudios de ecología de ríos habitualmente no se clasifican al mismo nivel), (apéndice 1 ).

Las listas de especies obtenidas para cada muestra se han estudiado y comparado según los distintos criterios. La relación entre especies se estudió cuantificándola con un índice de asociación, la correlación de rango.

Las comparaciones entre muestras se han realizado utilizando dos medidas de distancia. Cuantitativamente se ha utilizado el error de la suma de cuadrados: $\mathrm{Ep}=1 / \mathrm{M}(\mathrm{Xij}-\mathrm{Vip})^{2}$ donde $\mathrm{Xij}$ es la abundancia de la especie «j» en la muestra «i», y V es la media de las abundancias de la especie «i» en el grupo P. Por otro lado se ha utilizado un índice de afinidad semi- 

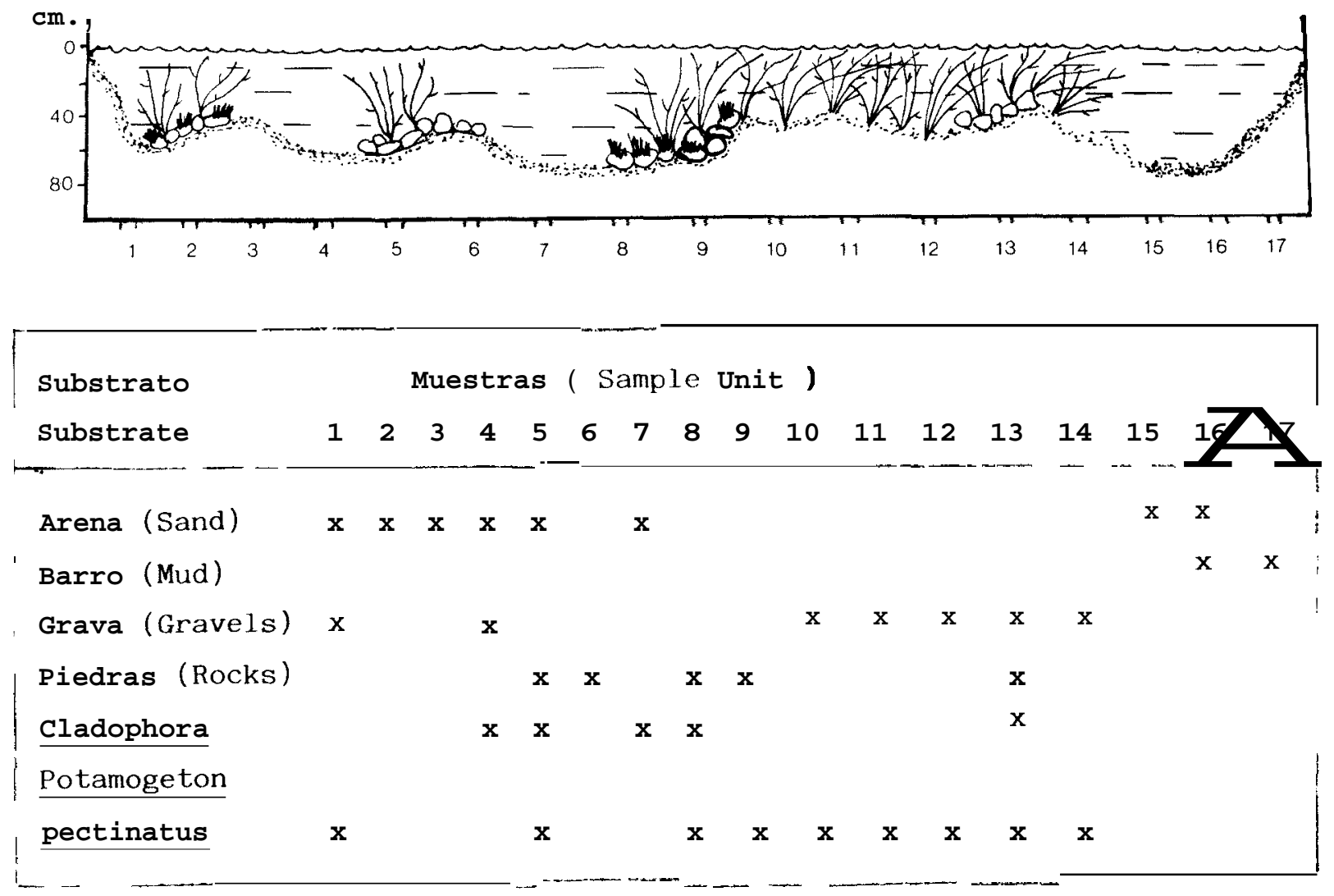

Figura 2.-Representaciónesquematizada del transecto realizado, indicando los tipos de sustrato y su distribución. Schematic representation of the transect showing the substrate type distribution.

métrico. el índice de Steinhaus: $D=1-S=2 W / A+B$, donde $\mathrm{W}$ es la abundancia mínima de las especies en las muestras que se comparan y A y B son los respectivos valores de abundancia para las distintas muestras. (Legendre \& Legendre, 1979).

Tanto la matriz de correlaciones de las especies como las de distancias entre muestras se ordenaron siguiendo un método jerárquico con el criterio de fusión de las medias. Las correlaciones cofeneticas obtenidas en cada una de las tres agrupaciones son superiores a 0,8 . Por otra parte aplicando el índice de Shannon-Weaver hemos estudiado cómo varía la diversidad a lo largo del transecto así como la forma del espectro al tomar un segmento mayor.

$\mathrm{La}$ heterogeneidad definida como $\mathrm{Z}=\mathrm{H}_{-}-[(\mathrm{Hi}-$ $\mathrm{Hi+1} / 2$ ] donde $\mathrm{H}_{i}=$ diversidad en la muestra «i» $\mathrm{y}$ $\mathbf{H}_{1}=$ la diversidad conjunta entre las dos muestras, se ha calculado para las muestras contiguas de la transección.

\section{RESULTADOS}

Del total de 100 taxones identificados en las 17 muestras los grupos mayoritarios los constituyen: Chironomidae (24), Oligochaeta (20), Ephemeroptera (17), Mollusca (7), Trichoptera (5).

- Dos terceras partes de los taxones identificados son especies con abundancias muy escasas. En estos casos sólo se detalla su presencia y abundancia total en las muestras (apéndice 1) y únicamente se han tenido en cuenta cuando se han comparado las muestras y se ha estudiado el transecto de forma secuencial, pero no cuando se han correlacionado las especies más abundantes.

\section{Asociación entre especies}

El tercio de especies más abundantes o cuando menos presentes en la mayoría de las muestras se han estudiado con más detalle, profundizando en su estructuración a nivel de comunidad. 
Las correlaciones de rango calculadas para estas especies (35) se han ordenado de forma jerárquica. El dendrograma resultante (figura 3 ) nos muestra 5 grupos de especies que sintetizan algunas de las características de la comunidad de macroinvertebrados ya que estos grupos responden a las características de las condiciones donde aparecen. La secuencia del 1 al 5 podría describirse como el paso de sustrato blando a macrófitos y de estos a cantos o piedras.

Los dos grupos que presentan las correlaciones más bajas son el 1 y el 5. Esto puede interpretarse como una inexistencia real (o falta de significado)de estos grupos (caso del grupo 1) o bien que son grupos donde van a parar todas aquellas especies poco correlacionadas con el resto (comoel grupo 5).

En efecto el grupo 1 está formado por Potamothrix, L. udekemianus, Polypedilum spp., Psychomyia y Synorthocladius semivirens. Estas especies se encuentran distribuidas más o menos por todas las muestras y su agrupación no ofrece ninguna explicación coherente.

En el caso del grupo 5 las especies que lo forman (Dina, Elmis, Ecdyonurus, Polycentropus), son especies que se presentan de forma poco abundante en las muestras. En general son especies que aparecen en las zonas reófilas del río Llobregat pero siempre en abundancias menores (Prat, et al.. 1983).

El grupo 4 es un grupo de especies propias de zonas de corriente, lo constituyen los Simúlidos, tres especies de Baetis, Hydropsyche exocellata y el molusco Ancylus. Todas estas especies son características de las zonas reófilas con sustrato formado por cantos.

El grupo 3 está formado mayoritariamente por Quironómidos (7 especies), 2 Moluscos: Bythinella y Potamopyrgus, y el oligoqueto Ophidonais. Esta última presenta sus valores máximos en abundancia en las muestras 12, 13 y 14. Estas muestras se obtuvieron de sustrato formado por Potamogeton y arena, lo que podría indicar la relación de este grupo con estos sustratos.

El grupo 2 lo constituyen especies de Oligoquetos (6) y el Quironómido Isocladius, ambas se pueden asociar a los sustratos blandos muestreados en la zona inicial y final de la transección (figura2).

\section{Comparación de las muestras}

En el estudio y comparación de las muestras hay que determinar con precisión el criterio de comparación. En nuestro caso partimos de la base que cada muestra es una identidad única.

Cuando la comparación se establece a nivel estrictamente cuantitativo usando el error en la suma de de cuadrados de las abundancias reales respecto a las medias de cada especie se obtiene una matriz de distancias. que agrupadas, (para simplificar la interpretación) nos muestra poca similaridad entre muestras (figura4a).
En efecto, el resultado que obtenemos de la comparación de las muestras según este criterio sólo nos permite identificar las muestras representativas de las zonas donde la fauna es más escasa. Como vemos en el dendrograrna (figura 4a) estas muestras son las número 1, 2, 3, 6 y 7 de la parte inicial de la transección y las 16 y 17 de la parte final.

En su mayoría son muestras de los extremos de la transección y forman un grupo que se asemejan entre sí más que cada una de las muestras restantes. Son todas las muestras propias de sustratos blandos (figura 2). Con este método el resto de las muestras no forman grupo alguno y se van concatenando secuencialmente (figura 4a) por lo que según este criterio las muestras no son semejantes. Esto nos dice que las muestras son poco parecidas tanto por la existencia de especies ocasionales de presencia desigual como por las marcadas fluctuaciones en la abundancia de las especies mayoritarias.

Si utilizamos otro criterio de comparación entre las muestras en el que no consideremos las dobles ausencias y la similaridad entre muestras es el cociente entre el valor mínimo de la abundancia de aquella especie en las muestras que se comparan (índice de Steinhaus) la agrupación varía. Como en el caso anterior se han ordenado y agrupado las similaridades obtenidas (figura4b).

En este caso las similaridades son altas entre muestras tanto con especies abundantes, muestras 10 y 14 o 5 y 9. como entre muestras pobres en individuos, la 4 y 7 (figura $4 b$ ).. El árbol jerárquico representa tres grupos principales. El grupo central está constituido por las muestras 5, 8, 9, 10,11, 12, 13 y 14. Todas estas muestras aparecían independientes cuando utilizábamos el criterio cuantitativo (compárese con la figura 4a). Son las muestras en las que las especies presentan abundancias mayores y las que se obtuvieron sobre sustratos más diversificados compuestos todos ellos por macrófitos y arena, o bien Cladophora y cantos de tamaño grande, o los dos tipos unidos. En cada una de las muestras el sustrato nunca era simple y en muchos casos estaba compuesto por más de dos de aquellos componentes (figura 2).

Los otros dos grupos están formados por las muestras $4,7,15$ y 16 y 1, 2, 3 y 6 respectivamente. La mayoría de estas muestras constituían el grupo de muestras de abundancias individuales bajas establecido con el método anterior.

En el grupo formado por las muestras 1, 2, 3 y 6 el sustrato muestreado era arena y sólo apa recía algo de Potamogeton en la muestra número 6. El grupo (4,7. 15 y 16) también son muestras recolectadas sobre sustrato blando, arena en las estaciones 4 y 7 y arena y barro en las muestras 15 y 16.

Algunas de las muestras a pesar de pertenecer a uno de estos dos grupos tienen valores de similaridad 


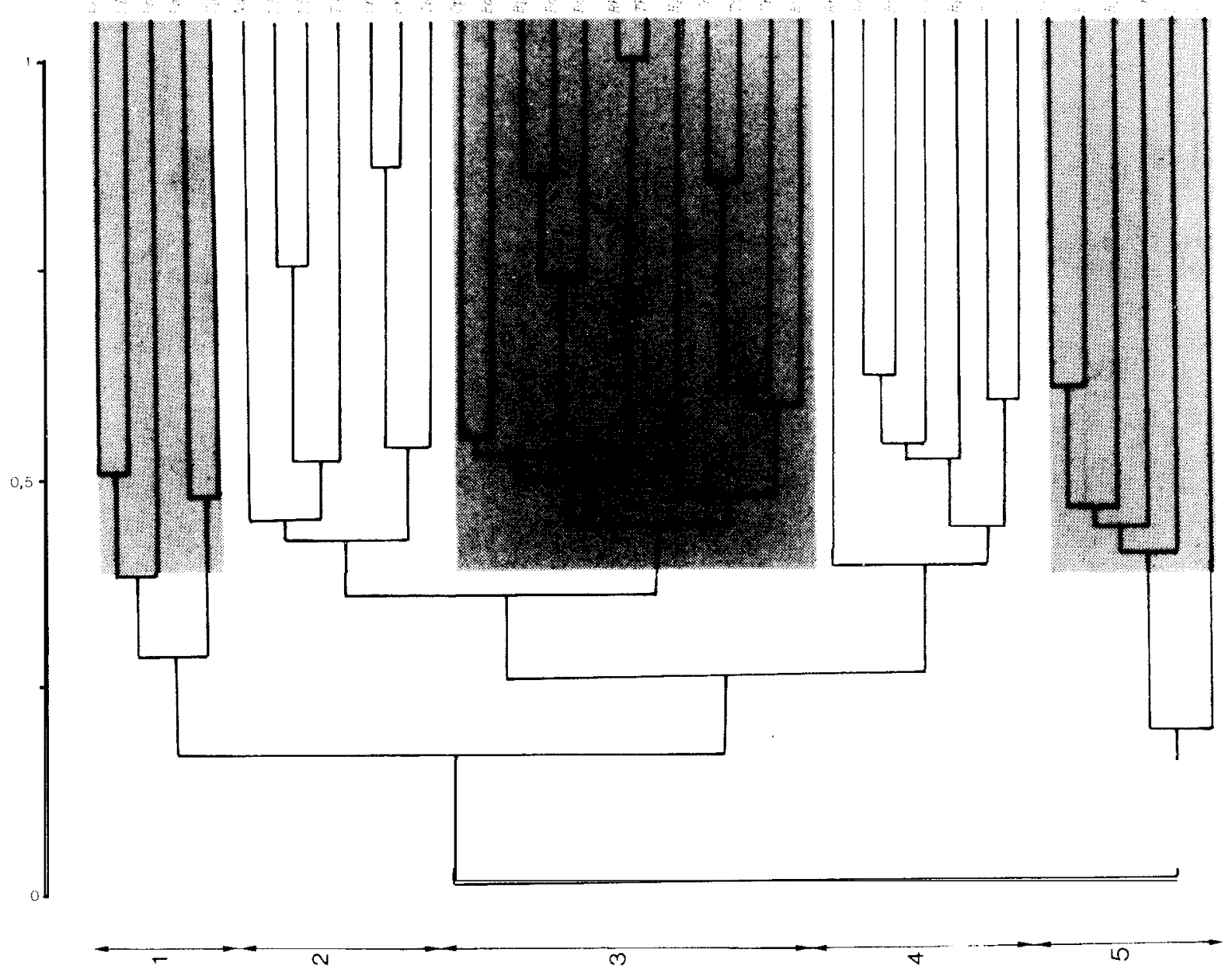

Figura 3.-Grupos de especies.

Species clustering. 

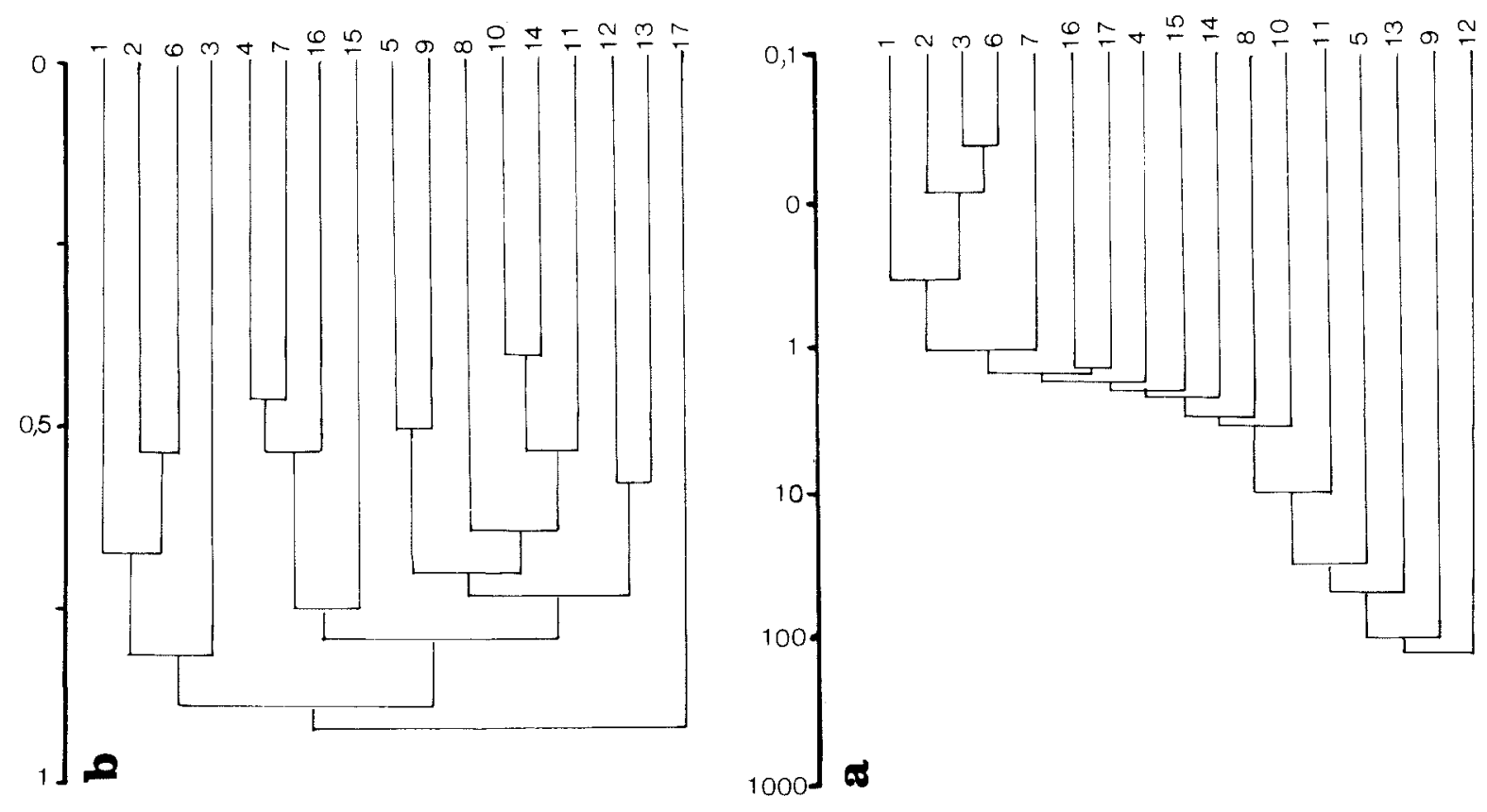

Figura 4.-Agrupación de las 17 muestras: a) Método cualitativo; b) Indice semimetnco.

Clustering of the 17 samples: a) Qualitative method (error of the sum of squares); b) Semimetric index (Steinhaus)

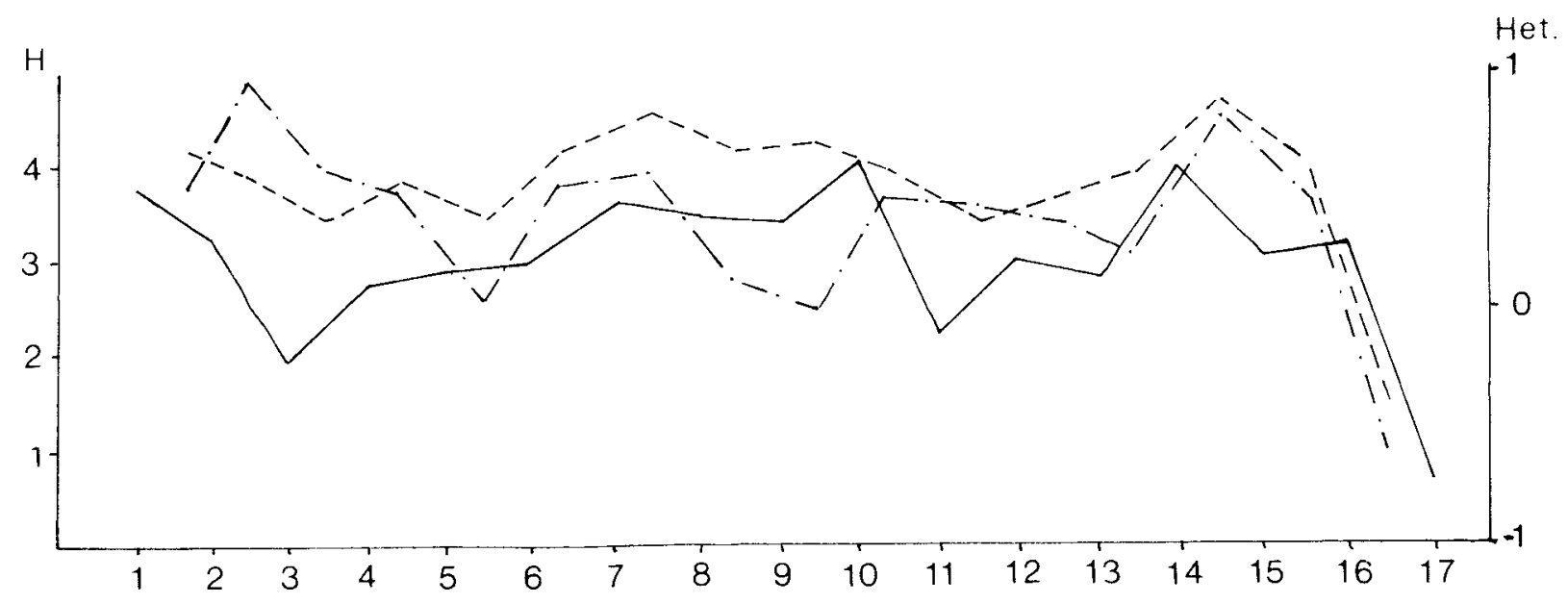

Figura 5.-Valores de diversidad (-)

Diversity (-).

Espectro de diversidad (--).

Diversity spectrum (--).

Heterogeneidad (-.-).

Feterogeneity (-.-). 
muy bajos. Por ejemplo este es el caso de las estaciones 3 y 17 que aparecen muy independientes de las otras muestras sobre sustrato blando o de la muestra 15 que se diferencia de las otras muestras reófilas.

\section{Diversidad}

Los valores de diversidad para cada una de las muestras (figura 5) sintetizan características como el n. ${ }^{\circ}$ y la abundancia de las especies.

Las muestras n. 10 y 14 tienen valores puntuales superiores a $4 \mathrm{Bit} /$ individuos. Ambas muestras están próximas a muestras con un $\mathrm{n}^{\circ}{ }^{\text {de }}$ individuos en algunas especies muy alto (muestra 13 y 9) y además situados en puntos donde apreciamos cambios en las condiciones. En el caso de la muestra n. 10 el cambio de sustrato respecto a la n. 9 (Ckadophora)es claro. En el caso de la niuestra n." 14 los cambios son tanto con la n. 15 (barro y tierra) así como con la n." 13 (piedras, Potamogeton, Cladophora). Así pues, estas dos muestras reflejan la zona de interfase donde se superponen las distribuciones de las especies con abundancias bajas en estas muestras y muy abundantes en alguna muestra próxima.

Las muestras con diversidades más bajas son la 17, 3 y 11 por este orden. La muestra n. ${ }^{\circ} 17$ está en el extremo, en una zona profunda donde el sustrato formado por barro y tierra presentaba un n. ${ }^{\circ}$ reducido de especies con abundancias bajas. La muestra n." 3 del extremo inicial se ha recogido en un sustrato de arena exclusivamente. La muestra 11 tiene un valor bajo, frutodc la presencia masiva de Ophidonais serpentina, que constituye el $50 \%$ de la muestra.

\section{Espectro de diversidad y heterogeneidad}

Al combinar las muestras contiguas y calcular las diversidades nuevamente obtenemos el espectro de diversidad, la interpretación del espectro permite dis. tinguir las zonas de cambio en la diversidad y las interfases o puntos de cambio de la comunidad.

Los picos en el espectro (Fig. 5) aparecen en las muestras iniciales 1 y 2, medias 7 y 8 y finales 14 y 15. El efecto «bien conocido». de los márgenes del río, se refleja en el pico inicial en el espectro. Seguidamente baja y se estabiliza en la l.a zona de tierra y Potarnogcton. Aparece una interfase, muestras 6 y 7 , para llegar a la muestra de sustrato duro 8, 9, 10 en el tramo de diversidad media mayor.

El paso a la zona de Potamogeton y tierra produce un nuevo descenso (figura 5 ) muestras $11,12,13,14$ y con valores similares a los de las muestras 3, 4, 5, 6 . El tercer pico lo produce el paso de la muestra de Potamogeton, al dominio de arena y fango (figura 5 ) muestras 15, 16, 17.

El cálculo de la heterogeneidad revela la zona donde la composición es más diversa. Los valores de heterogeneidad son altos para las primeras muestras y reflejan el efecto de la orilla (figura5). Se mantienen relativamente altas hasta la muestra n." 5. vuelve a crecer hasta la n. ${ }^{\circ}$. En la muestra 9 y 10 se vuelve a reducir. Crece en los valores de las siguientes muestras $(10,11)$ para disminuir progresivamente hasta la muestra n. 15 donde tenemos un pico máximo $(14$, 15) que se reduce algo en la siguiente $(15,16)$ y se hace negativa en la ultima pareja $(16,17)$.

\section{DISCUSION}

El estudio de 17 muestras secuenciales en la zona media del río Llobregat permite distinguir grupos de especies características preferentes de zonas donde las condiciones son distintas:

- especies asociadas a la vegetación macrofítica.

- especies presentes en sustratos blandos.

- especies reófilas de sustratos duros.

Existen otros grupos de especies que no se pueden asociar a ninguna condición del medio concreto y que han de responder a otros factores (grupo 5) o sencillamente que no están bien representados en el muestreo (grupo 1).

La complejidad que resumen cada unidad de muestr e se refleja en los resultados de las agrupaciones que se han obtenido. La clasificación de la muestra a que hemos llegado cuando se emplea un criterio cualitativo no describe la similaridad entre muestras con pocas especies e individuos, y las diferencias entre todas aquellas ricas en especies y número de individuos elevado.

El índice de asgciación semicuantitativo es mucho más revelador de la presencia de muestras con número de organismos y numero de especies elevados. Otros grupos formados con este índice resultan de la similaridad de las muestras en los extremos de la transección, las muestras del extremo anterior por un lado y las del extremo posterior por otro. Otro tipo de muestras son aquellas que no se identifican con ningún grupo y son las muestras pobres o poco representativas, consecuencia del método de muestreo utilizado. La convención establecida de obtener muestras cada $2 \mathrm{~m}$. provoca el muestreo de zonas pobres poco diferenciadas. Estas muestras poco reveladoras cuando el estudio se centra en la caracterización de las coniunidades, resultan ser todo lo contrario cuando estudiamos ! as variaciones espaciales.

Los valores de diversidad mayores aparecen en la zona del transecto en muestras contiguas a otras que se tomaron en una zona de condiciones muy heterogéneas. En estas muestras más diversas aparecen muchas especies y con abundancias bajas.

La consideración de las muestras secuencialmente permite distinguir zonas de condiciones más o menos uniformes con valores de diversidad media diferentes; zonas con macrófitos menos diversificados de las zonas con cantos y Cladophora. 

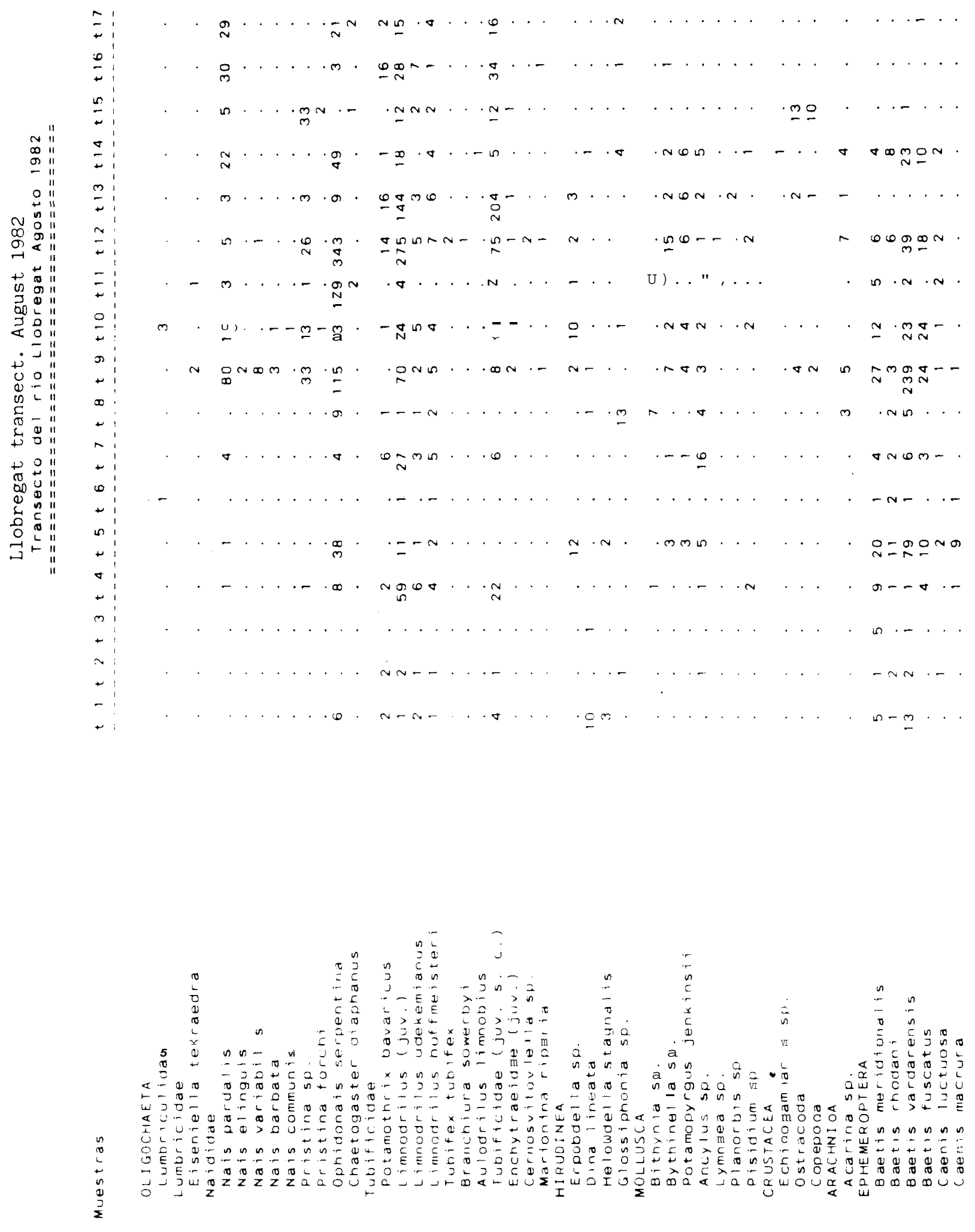
3
5
0
5
5
0
5
$m$
5
$\vdots$
5
5
5
5
$m$
+
$\infty$
+
5
+
0
5
5
+
+
+
5
+
+
+
5
+
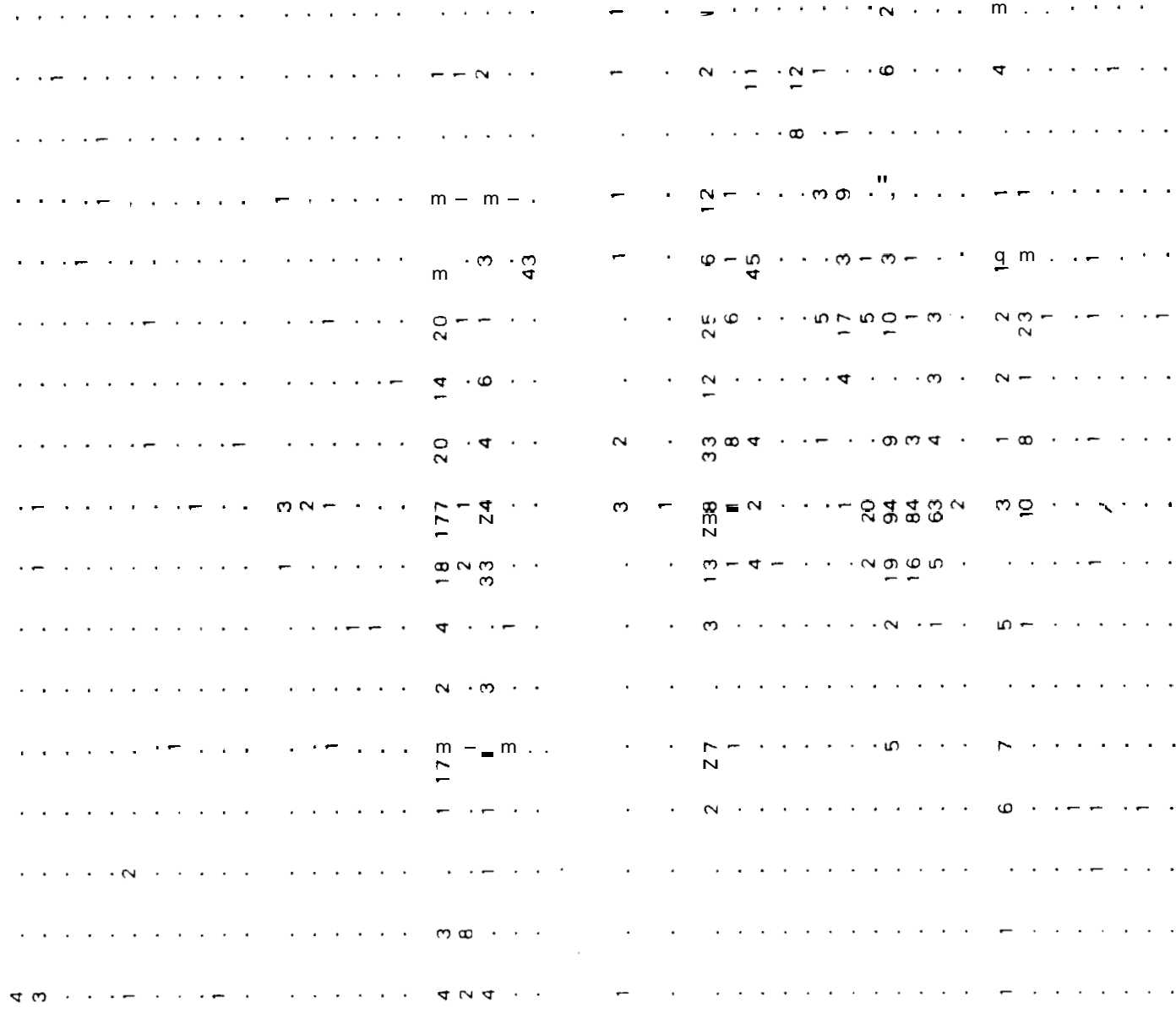

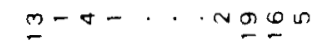


Llobregat transect. August 1982

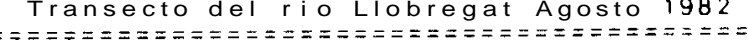

\section{Muestras}

Micropsectra atrofasciata Rheotanytarsus sp.
Simulidae

Simulium equinum

5. lat inum-rubzovi anum

Culicidae

Empididae (Atalantidae)

Ephydr-dae

Anthomyidae

Tipulidae

Dolichopodidae

Psycodidae

Ceratopogonidat t $1 \mathrm{t} 2 \mathrm{t} 3 \mathrm{t} 4 \mathrm{t} 5 \mathrm{t} 6 \mathrm{t} 7 \mathrm{t} 8 \mathrm{t} 9 \mathrm{t} 10 \mathrm{t} \mathrm{t} \mathrm{t} 12 \mathrm{t} 13 \mathrm{t} 14 \mathrm{t} 15 \mathrm{t} 16 \mathrm{t} 17$

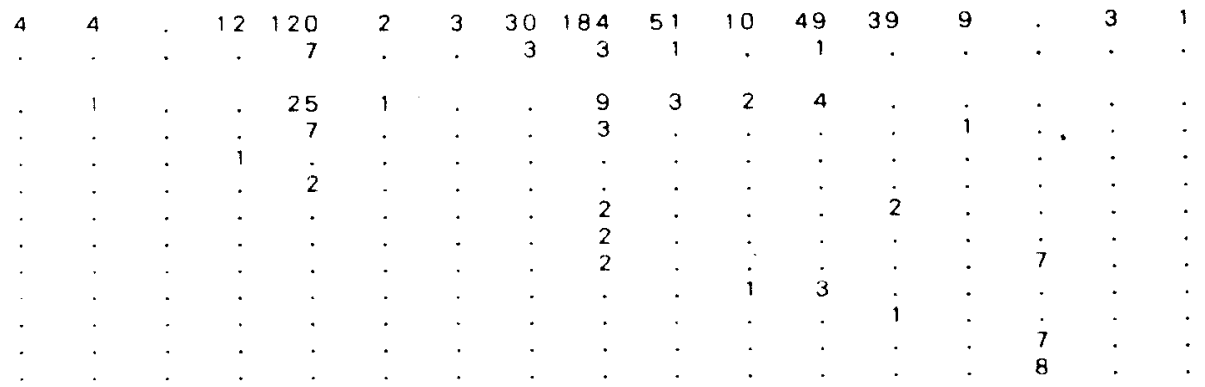


Por su parte la heterogeneidad se hace extrema en los márgenes del río y fluctúa dentro de estos márgenes a lo largo del transecto.

La utilización de un transecto permite abordar el estudio de la estructuración especial de las comunidades de macroinvertebrados en el lecho del río. Así mismo la secuencionalidad de las muestras hace posible descubrir la estructuración global del río en aquel punto.

El empleo de muestreos de este tipo permitiría contrastar la hipótesis que predice una reducción de la diversidad en los cauces aguas abajo (Vannote $e t$ al 1980). Esta reducción de la diversidad no tan clara para otros autores (Webster et al 1983) solo puede establecerse si no se considera la diversificación espacial que aquí hemos descrito.

\section{BIBLIOGRAFIA}

Legendre, L. et Legendre, P. (1979). Ecologie Numérique. Ed. Masson, Paris.

Millet, X.; Prat, N., 1984. Las comunidades de macroinvertebrados a lo largo del río Llobregat. Limnética (1): 222-233.

Prat, N.; Puig, M.A.; Gonzalez, G.; Tort, M.J. (1982). Predicció i control de la qualitat de les aigües dels rius Besós i Llobregat. I. Els factors fisics i quimics del medi. Estudis i Monografies No 6. Servei del Medi Ambient. Diputació de Barcelona.

Prat, \%.; Puig, M.A.; González, G. (1983). Predicció i control de la qualitat de les aigües dels rius Besós i Llobregat. 11. El poblament faunístic i la seva relació amb la qualitat de les aigües. Estudis $i$ Monografies N9 Servei del Medi Ambient. Diputació de Barcelona.

Prat, N.; Puig, M.A.; Gonzalez, G.; Millet, X. (1984). Chironomid longitudinal distribution and macroinvertebrate diversity along the Llobregat river (NE Spain). Mem. Amer. Ent. Soc: 34: 267-278.

Resh, V.H. (1957). The use of transect sampling in estimating single species production of aquatic insects. Verh. Internat. Verein. Limnol. 19: 3089-3094.

Resh, V.H. (1979). Sampling variability and life history features: basic considerations in the design of aquatic insect studies. J. Fish. Res. Bourd. Can. 36: 290-311.

Vannote, R.L.; Minshall, W.; Curnrnins, K.W.; Sedell, J.R.; and Cushing, C.E. (1980). The river continuum concept. Cun. J. Fish. Aquat. Sci. 37: 130-137. Webster, J.R.; Gurtz, M.E.; Hains, J.J.; Meyer, J.L.; Swank, W.T.; Waide, J.B.; and Wallance, J.B. (1983). Stability of Stream Ecosystems. In Stream Ecology. Ed. Barnes, J.R. and Minshall, G.W. 